\section{Gracias por todo: Una revisión sobre la gratitud desde la neurobiología a la clínica}

\author{
ÁLVARO TALA ${ }^{1}$
}

\section{Thanks for everything: a review on gratitude from neurobiology to clinic}

The World Health Organization (WHO) defines health not only as the absence of disease but as a state of complete physical, mental and social well-being. Gratitude is one of the concepts most commonly associated with well-being from the empirical point of view. Despite of this, we seldom use the concept, possibly due to the lack of dissemination of its scientific bases and possible clinical applications. At an anatomical level, it is related consistently with the medial prefrontal cortex and at molecular level, with the neuropeptide oxytocin. It has been associated with better physical and mental health parameters, both in healthy and sick populations. It is usually measured using scales, being the most used worldwide the Gratitude Questionnaire-6 item (GQ-6). There are exercises that can enhance gratitude and its benefits, such as the gratitude letter and the gratitude journal. Given the current state of knowledge about gratitude, and considering its potential benefits, low risks, ability to complement other treatments, the simple and cost-effectiveness of gratitude interventions, a continuing research on this topic and its practical implementation is warranted.

(Rev Med Chile 2019; 147: 755-761)

Key words: Emotions; Happiness; Health; Health Promotion; Psychology, Medical.
'Departamento de Psiquiatría Norte, Facultad de Medicina, Universidad de Chile. Santiago, Chile.

Esta investigación no contó con apoyo financiero.

Recibido el 28 de septiembre de 2018, aceptado el 4 de marzo de 2019.

Correspondencia a: Álvaro Tala Tajmuch Clínica Psiquiátrica Universitaria, Universidad de Chile. Av. La Paz 1003, Santiago, Chile. Teléfonos: +56229788601 o +56229788646 . alvarotalat@gmail.com

\section{L} a Organización Mundial de la Salud (WHO) define salud no solo como la ausencia de enfermedad, sino como un estado de completo bienestar físico, mental y social ${ }^{1}$. Pese a esto, el bienestar ha sido poco abordado por la medicina de forma empírica en comparación con el espacio dado al estudio de las patologías. En concordancia con la visión más amplia de la salud, en los últimos años ha habido un incremento sostenido de la evidencia respecto al hecho de que el bienestar y las emociones positivas no solo corresponderían a la contraparte de la enfermedad y de las emociones negativas, sino que tendrían una base neurobiológica y efectos en la salud de forma diferenciada, no siendo estos efectos explicables solo por la ausencia de enfermedad ${ }^{2}$.
En este sentido, la psicología positiva ha hecho grandes aportes, mostrando que el bienestar y sus componentes son susceptibles de ser intervenidos y potenciados, eventualmente aportando a una mejor salud y bienestar en general de las personas sanas y enfermas ${ }^{3,4}$.

En este contexto, el concepto de gratitud es de los que se ha asociado más robustamente al bienestar, por lo que la cantidad de estudios y de investigación empírica relacionada a este concepto ha aumentado significativamente en los últimos $a_{n} o^{5,6}$. Pese a esto, la evaluación e indicación de la gratitud en la práctica clínica no es habitual en Chile. Una posibilidad que pudiese explicar esto es la falta de conocimiento en términos científicos que pudiese existir en nuestro medio respecto de 
este concepto, por lo que impresiona relevante revisar el estado del conocimiento relacionado con la gratitud en aspectos que hagan más accessible su eventual incorporación a la clínica, como el concepto desde el punto de vista científico, su neurobiología, sus potenciales beneficios en el campo de la salud, su medición y su indicación.

\section{El concepto de gratitud}

Si bien el concepto se encuentra presente en todo el mundo y en todas las culturas, de una $\mathrm{u}$ otra forma, no se encuentra libre de controversia, existiendo diversos autores que lo han definido de diferentes maneras que pueden llegar a ser bastante disímiles, sin necesariamente ser dichas conceptualizaciones excluyentes entre $s^{6-8}$. Ha sido descrita como un estado emocional, como un rasgo, como una respuesta de afrontamiento, como una virtud moral, como un hábito, como una actitud y como una orientación de vida, sin embargo, las dos aproximaciones más frecuentemente utilizadas en investigación empírica son como rasgo o disposición y como estado afecti$\mathrm{vo}^{9,10}$. Como rasgo o disposición usualmente alude a la tendencia individual y generalizada a notar y experimentar aprecio por lo bueno en la vida, presentando aquellos con mayor gratitud disposicional una mayor tendencia a sentir gratitud con mayor frecuencia, intensidad y ante una mayor variedad de personas o estímulos ${ }^{8}$. Por su parte, la gratitud como estado emocional o afectivo alude a una sensación que surge cuando uno reconoce que ha obtenido un resultado valorado personalmente como positivo de una fuente externa ${ }^{7,10}$. Generalmente implica que la persona percibe que recibe un beneficio valioso, sobre todo si no fue buscado, merecido, ganado o secundario a intenciones ocultas de un otro, esto ya sea en relación a un acto de generosidad por un benefactor concreto, o por otras fuentes impersonales como podrían ser un evento, la naturaleza o Dios ${ }^{8,11,12}$.

\section{La neurobiología de la gratitud}

En general existen pocos estudios que aborden desde la perspectiva de la neurociencia la gratitud. La mayoría de los estudios existentes utilizaron como metodología de estudio resonancias magnéticas funcionales y con este método han asociado la gratitud a diferentes áreas cerebrales, las cuales pueden variar según se evalúe la gratitud como proceso afectivo, cognitivo, perceptivo o expresivo. Entre las áreas asociadas a la gratitud se han encontrado la corteza temporal superior derecha ${ }^{13}$, el giro temporal inferior derecho ${ }^{14}$, la corteza frontoparietal izquierda, la amígdala ${ }^{15} \mathrm{y}$ la corteza cingulada anterior ${ }^{16}$, entre otras áreas, sin embargo, la más consistentemente asociada ha sido corteza prefrontal medial ${ }^{13,16,17}$. Como soporte adicional al rol de esta última estructura en la gratitud, un estudio mostró que la práctica de ejercicios de gratitud puede inducir cambios en regiones de la corteza cerebral asociadas a recompensas cuyo valor depende del contexto, como la corteza prefrontal ventromedial, mientras que otro mostró que el uso de estimulación transcraneal con corriente continua en esta zona podría modificar los niveles de gratitud ${ }^{18,19}$. En este sentido, se comprende la gratitud como un fenómeno en el que participan diversas áreas cerebrales involucradas en el proceso de reconocer, interpretar, valorar y responder a determinados estímulos, tanto cognitivos como emocionales, ya sean internos o externos.

Desde el punto de vista electroencefalográfico, hasta el momento no se ha podido identificar un patrón que permita diferenciar claramente la gratitud de otras emociones como la esperanza, el orgullo y la inspiración ${ }^{20}$.

A nivel molecular se ha involucrado a los opiodes, las monoaminas y principalmente a la oxitocina. El primero es un sistema que se ha ligado tradicionalmente con el dolor, sin embargo, también se ha descrito que podría tener un rol en las relaciones sociales, el apego, la motivación y la gratitud, principalmente mediado por el receptor opiode $m u^{21}$. En cuanto a monoaminas, se encuentra descrito que un polimorfismo del gen de la catecol-O-metiltransferasa (COMT), enzima encargada de degradar monoaminas, podría explicar un porcentaje de las variaciones en los niveles de gratitud entre individuos ${ }^{22}$.

Finalmente, la oxitocina es un neuropéptido clásicamente involucrado en la modulación y procesamiento de conductas sociales, el cual se ha ligado a la gratitud mediante estudios que han asociado una mayor gratitud con polimorfismos del gen CD38, que regula los niveles de oxitocina $\mathrm{y}$ con polimorfismos de su receptor acoplado a 
proteína $G^{23,24}$. Esta relación entre oxitocina y gratitud también es reforzada por el hecho de que experimentalmente la administración intranasal de oxitocina ha producido aumentos en la gratitud $^{25,26}$.

\section{Potenciales beneficios de la gratitud en salud}

La gratitud se ha asociado a mejores parámetros en salud física como disminución de marcadores proinflamatorios y aumento de la variabilidad de la frecuencia cardiaca en reposo ${ }^{27}$, mejor funcionamiento cardiovascular ${ }^{28}$, mejor calidad de sueño y disminución de la presión arterial $^{29}$, mejor respuesta inmune, menores niveles de HbAlc y a una menor cantidad de síntomas físicos en general ${ }^{30}$.

Desde la perspectiva de la salud mental, se ha asociado a resultados como mayores niveles de emociones positivas, satisfacción vital y conductas prosociales ${ }^{7,31}$ y a menores niveles de estrés en embarazadas ${ }^{32}$, sintomatología depresiva ${ }^{8,31,33}$, angustia ante la muerte ${ }^{34}$, distorsiones cognitivas asociadas al peso y a la imagen corporal ${ }^{35}$, sintomatología asociada al estrés ${ }^{36}$, psicopatología en general $^{37}$, sintomatología ansiosa ${ }^{38}$, consumo de sustancias $^{39}$ y suicidalidad ${ }^{40}$. Además, se ha asociado a una mejor calidad de vida y a una mayor adherencia a tratamiento ${ }^{41}$. Incluso se ha visto que intervenciones centradas en la gratitud podrían ser utilizadas en pacientes suicidas, con resultados promisorios, al menos en el corto plazo, en parámetros tan relevantes en esta población como la desesperanza ${ }^{42}$. Finalmente, también se ha descrito que podría utilizarse como complemento a la psicoterapia, mejorando los resultados de esta ${ }^{43}$.

Desde la perspectiva de sus cercanos, los amigos y familiares tienden a reportar que quienes practican la gratitud se ven más felices, es más grato compartir con ellos y, en general, se les considera más optimistas, confiables y dispuestos a ayudar.

Además de los beneficios descritos anteriormente en población general y clínica, una revisión reciente, que incluyó más de 32 artículos, exploró la gratitud en pacientes, familiares de estos y en profesionales de la salud. Esta mostró que, en general, los pacientes y familiares de estos son agradecidos por los cuidados que entrega el equipo de salud, particularmente si fue en momentos vulnerables, si se mostró preocupación real por la persona o si respondió adecuadamente a una necesidad particular, destacando principalmente las habilidades interpersonales por sobre las técnicas en los equipos. También mostró que cuando los profesionales de la salud perciben la gratitud de sus pacientes y se sienten agradecidos, mejora su bienestar, disminuyen sus niveles de burnout, incrementa su motivación y se promueve el desarrollo de una práctica profesional más plena y reflexiva, entre otros beneficios ${ }^{10}$.

En relación a lo anterior, podemos dar cuenta de la importancia de promover gratitud en los equipos y en la atención de salud, lo cual se ha visto que es posible mediante intervenciones concretas, las que tendrían ventajas en su aplicación en profesionales de la salud respecto de otras intervenciones, como el poder ser autoaplicadas, demandar poco tiempo y no ser estigmatizantes ${ }^{33}$.

\section{La medición de la gratitud}

Se han desarrollado numerosos instrumentos para medir la gratitud dependiendo de cómo se le conceptualice (ej: como rasgo vs como afecto). Esto último no es menor, dado que la forma de conceptualización y de medición puede influir en las conclusiones de los estudios. Por ejemplo, se ha visto que los estudios que examinan la gratitud como un estado afectivo tienden a reportar tamaños de efecto más grandes que aquellos que la miden como rasgo, y aquellos que evalúan la gratitud en respuesta a un estímulo concreto tienden a mostrar un tamaño de efecto mayor que aquellos enfocados en la gratitud en general en cuanto a la apreciación de lo que es valorado en la vida ${ }^{7}$.

Para medir la gratitud, generalmente se utilizan escalas, siendo las más habitualmente utilizadas en investigación las siguientes: The Gratitude Resentment and Appreciation Scale (GRAT), el Gratitude Questionnaire-6 item (GQ-6) y The Gratitude Adjective Checklist (GAC). Estas contarían con buena confiabilidad según un metaanálisis reciente, aunque otros parámetros psicométricos podrían requerir de mayor evaluación ${ }^{44}$. Las 3 escalas son autoaplicadas, midiendo las primeras 2 la gratitud como variable disposicional, mientras que la tercera podría medirla como estado afectivo o como variable disposicional, según el intervalo de tiempo que se indique en las instrucciones ${ }^{45}$. 
La más usada en diferentes países y contextos es el GQ-6, el cual consta de 6 ítems en un formato de respuesta de tipo Likert, puede ser autoaplicado y se ha visto que su confiabilidad no se ve influida en relación a si es administrada online o por papel $^{44}$. Este, además, tendría la ventaja en nuestro medio de encontrarse validado para población chilena $^{46,47}$.

\section{La indicación de la gratitud}

Una primera pregunta a definir sería si la gratitud es potenciable y si existen intervenciones dirigidas a promover la gratitud que realmente sirvan más allá de los estudios aislados o de las percepciones de los involucrados. Teniendo en cuenta este punto, cabe destacar dos metaanálisis recientes, que mostraron que las intervenciones en gratitud podrían producir resultados favorables en cuanto a aumento del bienestar en sus distintos componentes, incluida la gratitud, y disminución del malestar psicológico, por ejemplo, de sintomatología depresiva, al menos de forma comparable a otras intervenciones que habitualmente requieren de mayores recursos, con tamaños de efecto al menos pequeños ${ }^{48,49}$. Esto requeriría de más y mejores estudios para poder afirmarse a ciencia cierta, sin embargo, considerando los potenciales beneficios, escasos riesgos, capacidad de complementar otros tratamientos, lo sencillo y lo costo-efectivo de las intervenciones, probablemente, la evidencia existente justifica considerar su posible utilización en clínica.

En cuanto a su mecanismo de acción, se ha planteado la hipótesis que su efecto en el bienestar podría estar relacionado con el hecho de que mejora el afrontamiento del estrés mediante el apoyo social, afrontamiento activo y el optimismo, disminuye la presencia de emociones negativas como la envidia, resentimiento, comparación social y el remordimiento, reduce el consumismo, aumenta el acceso a memorias positivas y podría aumentar el bienestar espiritual, entre otros mecanismos ${ }^{33}$. Se ha descrito también que el mecanismo por el cual la gratitud podría producir sus efectos a nivel de la salud física podría ser indirectamente a través de la reducción de otros elementos como el estrés y la sensación de soledad ${ }^{50}$.

La gratitud puede ser sentida, expresada o reforzada de formas muy diversas que pudie- sen servir como eventuales indicaciones, como palabras verbalizadas, escritas, abrazos, regalos y psicoeducación respecto del tema, entre otras formas ${ }^{6,8}$. Dentro de esta diversidad, las intervenciones más frecuentemente utilizadas en estudios empíricos son ${ }^{48}$ :

- Carta de gratitud: En esta actividad se escribe una carta a alguien para expresarle tu gratitud. Se han reportado variaciones con diferentes frecuencias y tiempos, siendo los más frecuentemente reportados desde 1 a 3 veces por semana dedicar 10 a $15 \mathrm{~min}$ a su escritura. La indicación habitualmente conlleva el escribir en términos específicos el por qué el paciente es agradecido con el sujeto escogido para la carta y cómo la conducta de este individuo lo afectó en su vida. También se ha reportado su redacción sin entregarla, con entrega presencial de la carta y su entrega de forma electrónica.

- Diario de gratitud: También llamado habitualmente conteo de bendiciones o lista de gratitud. En esta actividad se plantea el escribir un listado de elementos, situaciones o condiciones, ya sean cotidianas o no, por las que uno se siente agradecido. En algunas versiones, además, se indica proveer una explicación causal para cada evento. También en esta actividad se han reportado variaciones con diferentes frecuencias y longitudes de la lista, siendo los más frecuentemente reportados desde 1 a 3 veces por semana listas de 3 a 5 "bendiciones".

En cuanto a su efectividad, se ha visto que ambas intervenciones podrían ser eventualmente equivalentes $^{31}$. Pese a lo anterior, se debe considerar que se ha descrito que las personas tienden a preferir los diarios de gratitud, dado que la carta de gratitud cuenta con la posibilidad de un resultado negativo al entregar la carta, y en relación a esto genera una sensación de vulnerabilidad asociada a que, eventualmente, el bienestar personal depende de un otro y de su reacción, es decir, se percibe con un menor autocontrol y de un eventual costo mayor respecto del diario, lo que suele dificultar que las personas inicien y se comprometan con la actividad $^{51}$. Además, las personas tienden a subestimar los beneficios y sobreestimar las consecuencias negativas posibles de expresar su gratitud por este medio ${ }^{52}$.

En cuanto a la "dosis" de estos ejercicios, se ha visto que entre más tiempo se persista en la activi- 
dad, esta podría producir mayores efectos, por lo que los estudios suelen sugerir su realización durante varias semanas, sin embargo, la frecuencia, día, horario, longitud o tiempo concreto que debe dedicarse a cada una de las actividades han sido reportadas de forma variable en diversos estudios, sin contarse aún con alguna posología claramente superior a otra ${ }^{48}$. Pese a esto, se ha visto que para maximizar el efecto de las intervenciones en gratitud es recomendable que la persona se encuentre motivada a realizarla, realice una actividad con efectividad demostrada e, idealmente, vaya realizando variaciones a la actividad para disminuir la adaptación hedónica, por lo que probablemente la frecuencia, tiempo dedicado, longitud, horario $\mathrm{y}$ forma de la actividad deban ajustarse, al menos parcialmente, a cada persona ${ }^{53}$. Además, se ha visto que poblaciones específicas podrían obtener un mayor beneficio, como aquellas con mayores niveles de sintomatología depresiva y menores niveles de gratitud basales ${ }^{54,55}$.

En el entendido que toda intervención puede llegar a tener algún tipo de efecto adverso, las intervenciones en gratitud no son una excepción. En la literatura se ha descrito que en relación a la gratitud, la persona podría sentirse transitoriamente incómoda, culpable, avergonzada o en deuda ${ }^{56}$. Además, se ha descrito que en relación a sentirse agradecida en una situación injusta o perjudicial podría no realizar alguna acción correctiva pertinente ${ }^{6,57}$. Finalmente, se ha descrito también que los ejercicios de gratitud pudiesen estar contraindicados en pacientes con trastornos por uso de alcohol, bebedores frecuentes, cuyo objetivo terapéutico sea la abstinencia ${ }^{58}$.

\section{Consideraciones a futuro en torno a la gratitud y la clínica}

Aunque el estado actual del conocimiento en torno a la gratitud impresiona auspicioso, aún existen múltiples desafíos para su óptima implementación en la clínica. Por ejemplo, se debe determinar si hay algún momento, frecuencia, intensidad o variedad de intervención de gratitud que maximice sus beneficios, si existen umbrales definibles de exceso o déficit de gratitud, si existen intervenciones que pudiesen tener mejores resultados que las descritas, y cómo interactúan las intervenciones de gratitud con otras variables ${ }^{53}$.
También se ha visto que la percepción, la expresión y los beneficios que se pueden obtener de la práctica de la gratitud podrían variar en relación a aspectos como el género ${ }^{59}$, la etapa de la vida ${ }^{60}$, la cultura $^{61}$ y los niveles basales de psicopatología y de gratitud de las personas ${ }^{54}$. Estos desafíos, más que desalentar los avances en el campo de la gratitud y su uso clínico, debiesen ser un estímulo a realizar mayores estudios considerando lo costo-efectivas que son las intervenciones en gratitud y que, eventualmente, podrían potenciar los efectos de tratamientos ya existentes, desde la perspectiva del bienestar.

\section{Referencias}

1. Jeste DV, Palmer BW, Rettew DC, Boardman S. Positive psychiatry: Its time has come. J Clin Psychiatry 2015; 76 (6): 675-83.

2. Diener E, Pressman SD, Hunter J, Delgadillo-Chase D. If, Why, and When Subjective Well-Being Influences Health, and Future Needed Research. Appl Psychol Heal Well Being 2017; 9 (2): 133-67.

3. Bolier L, Haverman M, Westerhof GJ, Riper H, Smit F, Bohlmeijer E. Positive psychology interventions: A meta-analysis of randomized controlled studies. BMC Public Health 2013; 13 (1): 119.

4. Chakhssi F, Kraiss JT, Sommers-Spijkerman M, Bohlmeijer ET. The effect of positive psychology interventions on well-being and distress in clinical samples with psychiatric or somatic disorders: A systematic review and meta-analysis. BMC Psychiatry 2018; 18 (1): 1-17.

5. Gulliford L, Morgan B, Kristjánsson K. Recent Work on the Concept of Gratitude in Philosophy and Psychology. J Value Inquiry 2013; 47 (3): 285-317.

6. Wood AM, Froh JJ, Geraghty AWA. Gratitude and well-being: A review and theoretical integration. Clin Psychol Rev [Internet] 2010; 30 (7): 890-905.

7. Ma LK, Tunney RJ, Ferguson E. Does gratitude enhance prosociality?: A meta-analytic review. Psychol Bull 2017; 143 (6): 601-35.

8. Emmons RA, Stern R. Gratitude as a Psychotherapeutic Intervention. J Clin Psychol Sess 2013; 69 (8): 846-55.

9. Lambert NM, Graham SM, Fincham FD. A prototype analysis of gratitude: Varieties of gratitude experiences. Personal Soc Psychol Bull 2009; 35 (9): 1193-207.

10. Aparicio M, Centeno C, Robinson C, Arantzamendi M. Gratitude between patients and their families and health professionals: A scoping review. J Nurs Manag 2018; 00: $1-15$. 
11. McCullough ME, Emmons RA, Tsang JA. The grateful disposition: A conceptual and empirical topography. J Pers Soc Psychol 2002; 82 (1): 112-27.

12. Froh JJ, Sefick WJ, Emmons RA. Counting blessings in early adolescents: An experimental study of gratitude and subjective well-being. J Sch Psychol. 2008; 46 (2): 213-33.

13. Fox GR, Kaplan J, Damasio H, Damasio A. Neural correlates of gratitude. Front Psychol [Internet]. 2015; 6: 1491.

14. Zahn R, Garrido G, Moll J, Grafman J. Individual differences in posterior cortical volume correlate with proneness to pride and gratitude. Soc Cogn Affect Neurosci 2014; 9 (11): 1676-83.

15. Kyeong S, Kim J, Kim DJ, Kim HE, Kim JJ. Effects of gratitude meditation on neural network functional connectivity and brain-heart coupling. Sci Rep. 2017;7(1):115.

16. Kini P, Wong J, McInnis S, Gabana N, Brown JW. The effects of gratitude expression on neural activity. Neuroimage. 2016;128:1-10.

17. Yu H, Cai Q, Shen B, Gao X, Zhou X. Neural substrates and social consequences of interpersonal gratitude: Intention matters. Emotion. 2017;17(4):589-601.

18. Karns CM, Moore WE, Mayr U. The Cultivation of Pure Altruism via Gratitude: A Functional MRI Study of Change with Gratitude Practice. Front Hum Neurosci. 2017; 11: 599.

19. Wang P, Wang G, Niu X, Shang H, Li J. Effect of Transcranial Direct Current Stimulation of the Medial Prefrontal Cortex on the Gratitude of Individuals with Heterogeneous Ability in an Experimental Labor Market. Front Behav Neurosci [Internet] 2017; 11: 1-9.

20. Hu X, Yu J, Song M, Yu C, Wang F, Sun P, et al. EEG Correlates of Ten Positive Emotions. Front Hum Neurosci [Internet] 2017; 11: 26.

21. Henning M, Fox GR, Kaplan J, Damasio H, Damasio A. A potential role for mu-opioids in mediating the positive effects of gratitude. Front Psychol. 2017;8:1-6.

22. Liu J, Gong P, Gao X, Zhou X. The association between well-being and the COMT gene: Dispositional gratitude and forgiveness as mediators. J Affect Disord [Internet] 2017; 214: 115-21.

23. Algoe SB, Way BM. Evidence for a role of the oxytocin system, indexed by genetic variation in $\mathrm{cd} 38$, in the social bonding effects of expressed gratitude. Soc Cogn Affect Neurosci 2013; 9 (12): 1855-61.

24. vanOyen Witvliet C, Root Luna L, VanderStoep J V., Vlisides-Henry RD, Gonzalez T, Griffin GD. OXTR rs53576 genotype and gender predict trait gratitude. J Posit Psychol [Internet] 2018; 9760: 1-10.
25. Barraza JA, Grewal NS, Ropacki S, Perez P, Gonzalez A, Zak PJ. Effects of a 10-day oxytocin trial in older adults on health and well-being. Exp Clin Psychopharmacol 2013; 21 (2): 85-92.

26. Human LJ, Woolley JD, Mendes WB. Effects of Oxytocin Administration on Receiving Help. Emotion 2018; 18 (7): 980-88.

27. Redwine L, Henry BL, Pung MA, Wilson K, Chinh K, Knight B, et al. A pilot randomized study of a gratitude journaling intervention on HRV and inflammatory biomarkers in Stage B heart faillure patients. Psychosom Med 2016; 78 (6): 667-76.

28. Mills PJ, Redwine L, Wilson K, Pung MA, Chinh K, Greenberg BH, et al. The role of gratitude in spiritual well-being in asymptomatic heart failure patients. Spiritual Clin Pract 2015; 2 (1): 5-17.

29. Jackowska M, Brown J, Steptoe A. The impact of a brief gratitude intervention on subjective well- being, biology and sleep 2015; 21 (10): 2207-17

30. Krause N, Emmons RA, Ironson G, Hill PC. General feelings of gratitude, gratitude to god, and hemoglobin Alc: Exploring variations by gender. J Posit Psychol [Internet] 2017; 12 (6): 639-50.

31. O'Connell BH, O'Shea D, Gallagher S. Feeling Thanks and Saying Thanks: A Randomized Controlled Trial Examining If and How Socially Oriented Gratitude Journals Work. J Clin Psychol 2017; 73 (10): 1280-300.

32. Matvienko-Sikar K, Dockray S. Effects of a novel positive psychological intervention on prenatal stress and well-being: A pilot randomised controlled trial. Women and Birth [Internet] 2017; 30 (2): e111-8.

33. Cheng S, Tsui PK, Lam JHM. Improving Mental Health in Health Care Practitioners : Randomized Controlled Trial of a Gratitude Intervention. J Consult Clin Psychol 2015; 83 (1): 177-86.

34. Cheng RWLLS. Gratitude lessens death anxiety. Eur J Ageing 2011; 8: 169-75.

35. Dunaev J, Markey CH, Brochu PM. An attitude of gratitude: The effects of body-focused gratitude on weight bias internalization and body image. Body Image [Internet] 2018; 25: 9-13.

36. Greene N, McGovern K. Gratitude, psychological well-being, and perceptions of posttraumatic growth in adults who lost a parent in childhood. Death Stud [Internet] 2017; 41 (7): 436-46.

37. Jans-Beken L, Lataster J, Peels D, Lechner L, Jacobs N. Gratitude, Psychopathology and Subjective Well-Being: Results from a 7.5-Month Prospective General Population Study. J Happiness Stud 2018; 19 (6): 167389.

38. Althaus B, Borasio GD, Bernard M. Gratitude at the End 
of Life: A Promising Lead for Palliative Care. J Palliat Med [Internet] 2018; 21 (11): 1566-72.

39. Leung CC, Tong EMW. Gratitude and Drug Misuse: Role of Coping as Mediator. Subst Use Misuse [Internet] 2017; 52 (14): 1832-9.

40. Li D, Zhang W, Li X, Li N, Ye B. Gratitude and suicidal ideation and suicide attempts among Chinese Adolescents: Direct, mediated, and moderated effects. J Adolesc [Internet] 2012; 35 (1): 55-66.

41. Millstein RA, Celano CM, Beale EE, Beach SR, Suarez L, Belcher AM, et al. The effects of optimism and gratitude on adherence, functioning and mental health following an acute coronary syndrome. Gen Hosp Psychiatry [Internet] 2016; 43: 17-22.

42. Huffman JC, DuBois CM, Healy BC, Boehm JK, Kashdan TB, Celano CM, et al. Feasibility and utility of positive psychology exercises for suicidal inpatients. Gen Hosp Psychiatry 2014; 36: 88-94.

43. Wong YJ, Owen J, Gabana NT, Brown JW, McInnis $\mathrm{S}$, Toth $\mathrm{P}$, et al. Does gratitude writing improve the mental health of psychotherapy clients? Evidence from a randomized controlled trial. Psychother Res 2018; 28 (2): 192-202.

44. Card NA. Meta-analyses of the reliabilities of four measures of gratitude. J Posit Psychol 2018; 1-11.

45. Froh JJ, Fan J, Emmons RA, Bono G, Huebner ES, Watkins P. Measuring Gratitude in Youth: Assessing the Psychometric Properties of Adult Gratitude Scales in Children and Adolescents. Psychol Assess 2011; 23 (2): 311-24.

46. Langer ÁI, Ulloa VG, Aguilar-parra JM, Araya-véliz C, Brito G. Validation of a Spanish translation of the Gratitude Questionnaire ( GQ-6) with a Chilean sample of adults and high schoolers. Health Qual Life Outcomes [Internet] 2016; 14 (1): 1-9.

47. Carmona-Halty MA, Marín-Gutierrez M, Belmar-Saavedra F. Análisis psicométrico del gratitude questionnaire 6 (GQ-6) en población chilena. Univ Psychol 2015; 14 (3): 865-72.

48. Davis DE, Choe E, Meyers J, Wade N, Varjas K, Gifford A, et al. Thankful for the little things: A meta-analysis of gratitude interventions. J Couns Psychol 2016; 63 (1): 20-31.

49. Dickens LR. Using Gratitude to Promote Positive Change: A Series of Meta-Analyses Investigating the Effectiveness of Gratitude Interventions Investigating the Effectiveness of Gratitude Interventions. Basic Appl
Soc Psych [Internet] 2017; 39 (4): 193-208.

50. O'Connell BH, Killeen-Byrt M. Psychosocial health mediates the gratitude-physical health link. Psychol Heal Med [Internet] 2018; 1-6.

51. Kaczmarek LD, Kashdan TB, Drazkowski D, Enko J, Kosakowski M, Szäefer A, et al. Why do people prefer gratitude journaling over gratitude letters? The influence of individual differences in motivation and personality on web-based interventions. Pers Individ Dif 2015; 75: 1-6.

52. Kumar A, Epley N. Undervaluing Gratitude: Expressers Misunderstand the Consequences of Showing Appreciation. Psychol Sci 2018; 1-13.

53. Lyubomirsky S, Dickerhoof R, Boehm JK, Sheldon KM. Becoming happier takes both a will and a proper way: An experimental longitudinal intervention to boost well-being. Emotion 2011; 11 (2): 391-402.

54. Harbaugh CN, Vasey MW. When do people benefit from gratitude practice? J Posit Psychol [Internet] 2014; 9 (6): 535-46.

55. Kaczmarek LD, Kashdan TB, Kleiman EM, Baczkowski B, Enko J, Siebers A, et al. Who self-initiates gratitude interventions in daily life? An examination of intentions, curiosity, depressive symptoms, and life satisfaction. Pers Individ Dif [Internet] 2013; 55 (7): 805-10.

56. Layous K, Sweeny K, Armenta C, Na S, Choi I, Lyubomirsky $\mathrm{S}$. The proximal experience of gratitude. PLoS One 2017; 12 (7): 1-26.

57. Wood AM, Emmons RA, Algoe SB, Froh JJ, Lambert NM, Watkins P. A Dark Side of Gratitude? Distinguishing between Beneficial Gratitude and its Harmful Impostors for the Positive Clinical Psychology of Gratitude and Well-Being. Wiley Handb Posit Clin Psychol 2016; 137-51.

58. Krentzman AR. Gratitude, abstinence, and alcohol use disorders: Report of a preliminary finding. J Subst Abuse Treat [Internet] 2017; 78: 30-6.

59. Kashdan TB, Mishra A, Breen WE, Froh JJ. Gender differences in gratitude: Examining appraisals, narratives, the willingness to express emotions, and changes in psychological needs. J Pers 2009; 77 (3): 691-730.

60. Allemand M, Hill PL. Gratitude From Early Adulthood to Old Age. J Pers 2016; 84 (1): 21-35.

61. Floyd S, Rossi G, Baranova J, Blythe J, Dingemanse M, Kendrick KH, et al. Universals and cultural diversity in the expression of gratitude. R Soc Open Sci 2018; 5 (5): 180391. 